\title{
Conductivity-type anisotropy in molecular solids
}

\author{
J. R. Ostrick, A. Dodabalapur, L. Torsi, A, J. Lovinger, E. W. Kwock, \\ T. M. Miller, M. Galvin, Magnus Berggren and H. E. Katz
}

\section{Linköping University Post Print}

N.B.: When citing this work, cite the original article.

Original Publication:

J. R. Ostrick, A. Dodabalapur, L. Torsi, A, J. Lovinger, E. W. Kwock, T. M. Miller, M. Galvin, Magnus Berggren and H. E. Katz, Conductivity-type anisotropy in molecular solids, 1997, Journal of Applied Physics, (81), 10, 6804-6808.

http://dx.doi.org/10.1063/1.365238

Copyright: American Institute of Physics (AIP) http://www.aip.org/

Postprint available at: Linköping University Electronic Press

http://urn.kb.se/resolve?urn=urn:nbn:se:liu:diva-74920 


\title{
Conductivity-type anisotropy in molecular solids
}

\author{
J. R. Ostrick, ${ }^{\text {a) }}$ A. Dodabalapur, ${ }^{\text {b) }}$ L. Torsi, ${ }^{c}{ }^{\text {A }}$ A. J. Lovinger, E. W. Kwock, T. M. Miller, ${ }^{\text {d) }}$ \\ M. Galvin, M. Berggren, and H. E. Katz \\ Bell Laboratories, Lucent Technologies, 700 Mountain Avenue, Murray Hill, New Jersey 07974
}

(Received 16 October 1996; accepted for publication 12 February 1997)

\begin{abstract}
Thin polycrystalline films of perylenetetracarboxylic dianyhydride (PTCDA), an organic molecular solid, exhibits substantial anisotropies in its electronic transport properties. Only electrons transport in the directions along molecular planes, while mainly holes transport in the direction normal to molecular planes. A series of measurements on both field effect transistors with PTCDA active layers and light emitting diodes with PTCDA transport layers documents the anisotropy seen in the electronic transport in thin films of PTCDA. (C) 1997 American Institute of Physics.
\end{abstract}

[S0021-8979(97)05910-0]

\section{INTRODUCTION}

Sublimed molecular solids such as $\alpha$-sexithiophene ( $\alpha$ 6T) and perylenetetracarboxylic dianyhydride (PTCDA) have interesting transport and optical properties which stem from their strong tendency to form polycrystalline films in which the molecular planes orient in fixed directions with respect to the substrate. For example, $\alpha$-6T forms films in which the individual molecules lie almost edge-on on most substrates. The hole mobility in a direction parallel to the substrate is relatively high $\left(0.01-0.1 \mathrm{~cm}^{2} / \mathrm{V} \mathrm{s}\right)$, and has led to the demonstration of field effect transistor action. ${ }^{1}$ Although individual molecules in molecular solids interact through weak Van der Waals forces, many such materials exhibit significant anisotropies in the magnitude of charge mobility with respect to transport direction along the crystal. ${ }^{2-5}$

Highly purified single crystals of molecular solids such as anthracene and naphthalene generally transport both electrons and holes but with different mobilities which depend on the direction of transport. ${ }^{4}$ On the other hand, thin polycrystalline films of many molecular solids (such as $\alpha-6 \mathrm{I}$ ) appear to transport only one carrier type. Transport in such films depends on a number of factors such as molecular orientation, electronic overlap, molecular orbital energy levels, traps, and sometimes the nature of the ambient. In this article, we evaluate some of characteristics of charge transport in thin films of PTCDA, which is shown to possess anisotropies in the species of charge that can be transported along different directions. We show that PTCDA transports electrons in the directions parallel to the molecular planes and mainly holes in the direction perpendicular to the molecular planes. The charge carriers are referred to as electrons and holes for convenience although it is recognized that in many such materials the carriers are polaronic in nature. ${ }^{6}$ The anisotropy in carrier type was deduced from a series of device based measurements. Field-effect transistor (FET) structures were used to study electronic transport along molecular

\footnotetext{
${ }^{a)}$ Present address: Department of Physics, University of California at San Diego.

${ }^{b}$ Corresponding author. Electronic mail: ananth@physics.lucent.com

${ }^{c}$ Present address: Department of Chemistry, University of Bari, Bari (Italy).

${ }^{d)}$ Present address: CPS Chemical Company, Old Bridge, NJ 08857.
}

planes at different temperatures. A series of multilayer light emitting diodes based on the well understood 8-hydroxyquinoline aluminum (Alq)/triphenyl diamine (TAD) material system ${ }^{7}$ were employed to study transport properties normal to the molecular planes.

\section{EXPERIMENTAL RESULTS AND DISCUSSION}

\section{A. Field effect transistors}

The FETs were fabricated by vapor depositing vacuum purified PTCDA from a baffled Mo crucible in a high vacuum deposition chamber $\left(<7 \times 10^{-6}\right.$ Torr $)$ on to prepatterned substrates as illustrated in the inset of Fig. 1. The light emitting diode (LED) structures were similarly made by deposition of various organic materials from Mo crucibles in a high vacuum deposition chamber. Commercially available high purity Alq and TAD were used in the light emitting diode structures.

The PTCDA molecules stack in planes nearly parallel to the substrate ${ }^{9,10}$ in both the LED and FET device structures. The molecular orientation and its measurements are discussed in more detail later in this article. Field-effect transistors measure electronic transport along the PTCDA active layer/gate dielectric interface; therefore, the measured electronic transport represents transport parallel to the molecular planes of PTCDA. Measurements in a vacuum probe station were made on transistors of channel lengths $1.5,4,12$, and $25 \mu \mathrm{m}$ with a channel width of $250 \mu \mathrm{m}$, and about $50 \mathrm{~nm}$ thickness of PTCDA on both $\mathrm{SiO}_{2} / \mathrm{Si}$ and $\mathrm{MgF}_{2} / \mathrm{Si}$ substrates. In Fig. 1, the drain-source current versus drain-source voltage for different gate voltages is shown for a device with a 12- $\mu$ m-long channel on a $\mathrm{SiO}_{2} / \mathrm{Si}$ substrate. The increasing drain-source current with positive gate voltage is characteristic of an $n$-channel FET. This indicates that an accumulation layer of electrons forms in PTCDA, and these electrons move along the PTCDA/gate dielectric interface. The measured carrier mobilities range from $10^{-5}$ to $10^{-4} \mathrm{~cm}^{2} / \mathrm{V} \mathrm{s}$. The transistors do not exhibit any inversion-mode ( $p$ channel) operation and a negative gate voltage results in further depletion of carriers. Furthermore, a careful examination of the current-voltage characteristics shows no evidence of hole transport ${ }^{11}$ even at electric fields of $2.5 \times 10^{5} \mathrm{~V} / \mathrm{cm}$. This is significant because the inability to realize $p$-channel 


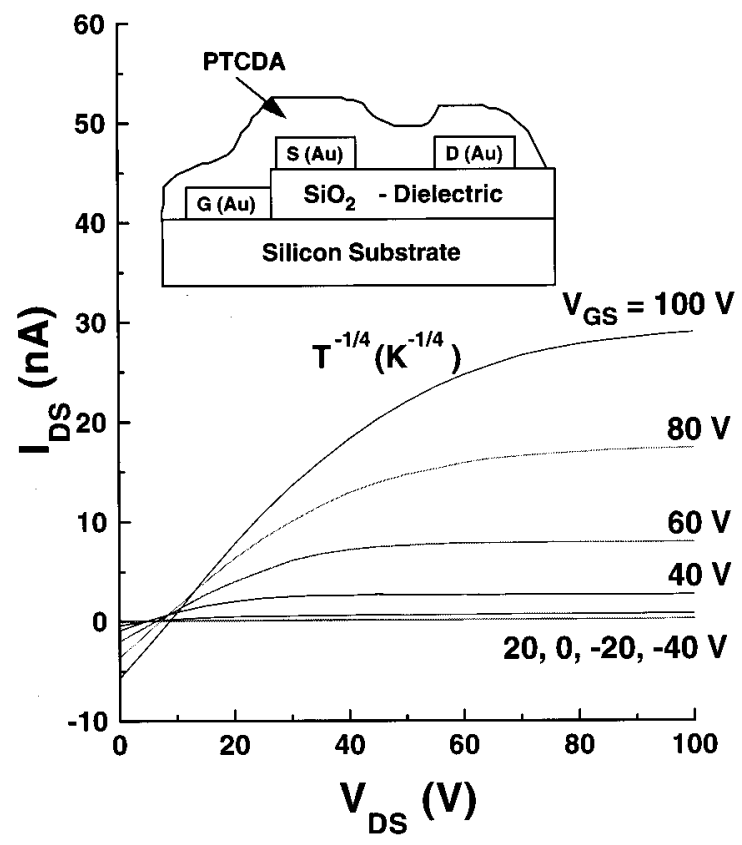

FIG. 1. Room temperature PTCDA field-effect transistor drain current as a function of drain-source voltage for a series of gate voltages. Positive gate voltages induce electrons in the channel resulting in an increase of the drain current with gate voltage. In the inset is shown the schematic structure of the field-effect transistors.

operation (i.e., a hole accumulation layer) may be because of difficulty in modulating the surface potential in a FET; however, the complete absence of a hole current points to a more intrinsic phenomena. This conclusion was corroborated by studying the characteristics of devices in which $\mathrm{MgF}_{2}$ is used in lieu of $\mathrm{SiO}_{2}$ as a gate dielectric. Similar behavior is seen in these devices. The FETs characteristics were also measured at temperatures ranging from 4.2 to $340 \mathrm{~K}$. PTCDA transistors do not exhibit field effect behavior for temperatures lower than 130 Kelvin. No evidence of any hole transport was found at low temperatures as well. A study of the variation of the temperature dependence of the field-effect mobility indicates that the carriers move by hopping. The very low mobilities are indicative of a very small electronic orbital overlap for electron transport in the direction of transport.

The field-induced carrier in the FET are located near the interface with the gate dielectric. Experimental work as well as modeling has shown that, when such FETs are operated in the accumulation mode, most of the field-induced carriers are located within $5 \mathrm{~nm}$ of this interface. This is because of the potential well created by the gate bias. The FET measurements therefore probe transport in this thin layer along the plane of the substrate.

It is important to note that the PTCDA FETs do not operate in moist air; however, under vacuum or at atmospheric pressure in an ambient of dry oxygen they exhibited $n$-channel FET behavior with the mobilities and electrical characteristics described above. The FETs did not function in a $\mathrm{N}_{2}$ ambient when the gas was bubbled through a water bath, indicating that moisture adversely affects electron transport in this material while oxygen does not appear to do so. Many electron transporting organic materials such as a

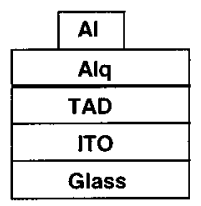

b

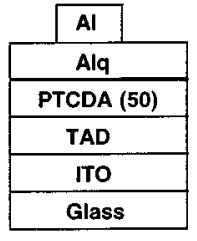

C

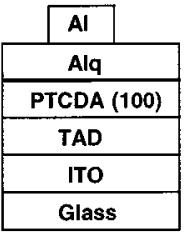

d

\begin{tabular}{|c|}
\hline Al \\
\hline Alq \\
\hline PTCDA (100) \\
\hline Alq \\
\hline TAD \\
\hline ITO \\
\hline Glass \\
\hline
\end{tabular}
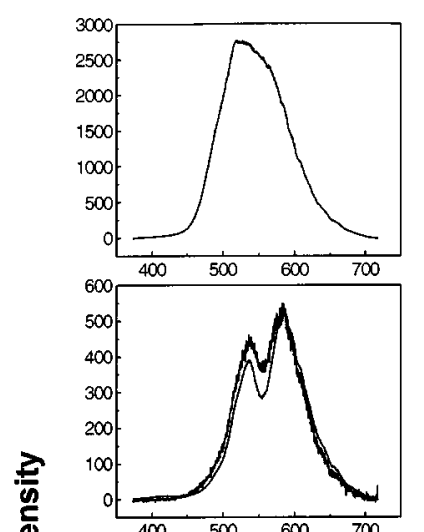

트
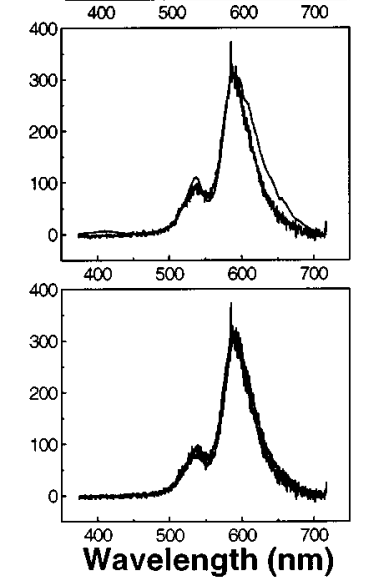

FIG. 2. (a) Layer structure and electroluminescence spectrum of an Alq (60 $\mathrm{nm})$ TAD $(60 \mathrm{~nm})$ LED. The emitted light originates from the Alq and is observed through the glass. (b) In this structure, a $50 \mathrm{~nm}$ PTCDA layer is placed between the TAD and Alq. The observed emitted light (solid line) still originates from the Alq but the spectrum is modified by partial absorption in the PTCDA layer. Also shown (dotted line) is the calculated spectrum. It will be easily recognized that for Alq to emit light in this structure, holes must be transported through the PTCDA. (c) Similar device structure as (b) but with $100 \mathrm{~nm}$ of PTCDA. (d) This LED has two Alq layers, one on each side of the PTCDA layer. The emission spectrum is identical to that of device (c).

$C_{60}$, naphthalenetetracarboxylic dianhydride are similarly affected by moisture. Interestingly, after exposure to moisture, the PTCDA FETs recover their electrical characteristics when tested under vacuum. This behavior was repeatedly reversible.

\section{B. Light-emitting diodes}

Pioneering work by Forrest and co-workers on contact barrier diodes $^{11,12}$ and LEDs $^{13}$ have clearly shown that PTCDA can transport holes normal to the molecular planes. Forrest et al. have also documented the huge anisotropies in the optical and dielectric properties of PTCDA along different directions. ${ }^{14}$ Additionally PTCDA has been shown to be a very good optical waveguide material, possessing a large refractive index (2.0) and low optical loss at near-infrared wavelengths along the molecular planes. ${ }^{15}$ Similar findings have been made subsequently by Ammermann and co-workers ${ }^{16}$ and Fuchigami et al. ${ }^{17}$

We have fabricated a series of multilayer LED structures in order to reconfirm hole transport normal to the molecular 


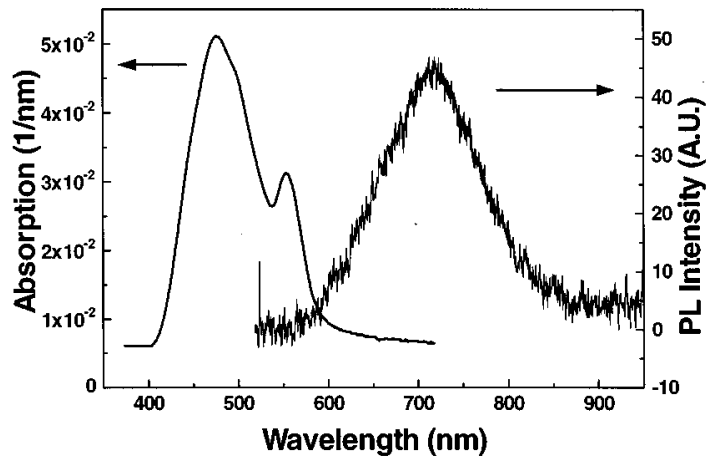

FIG. 3. The absorption and photoluminescence spectra of PTCDA.

planes and further test the ability of PTCDA to transport electrons in this direction. The LED structures are based on the TAD/Alq material system [Fig. 2(a)] in which the TAD solely transports holes ${ }^{7}$ and the Alq functions as a lightemitting layer. Our previous work with TAD/Alq LEDs, as well as recent results ${ }^{18}$ show that Alq has the ability to transport holes in addition to electrons. The device structures have been designed so as to provide a single PTCDA layer the opportunity to transport(i) holes [Figs. 2(b) and 2(c)], (ii) either electrons or holes [Fig. 2(d)], and (iii) electrons (not shown). All of the LED electroluminescent spectra was measured in air. The absorption spectrum of PTCDA (Fig. 3) has a local maximum near $550 \mathrm{~nm}$ which will cause a doublehump structure in the electroluminescence (EL) spectra when light from the Alq layer passes through the PTCDA layer. By varying the thickness of the PTCDA layer, the EL spectra will offer us a means to identify the source of light and to test the transport properties of PTCDA.

The PTCDA absorption spectrum was determined with a spectrophotometer and is consistent with other published reports. ${ }^{12}$ We also measured the photoluminescence spectrum of PTCDA, as shown in Fig. 3, with excitation from a multiwavelength Ar laser. It is important to note that photoluminescence from PTCDA is spread over a substantially different range of wavelengths than luminescence from Alq.

A layer of PTCDA is placed between Alq and TAD in order to test the ability of PTCDA to transport holes. Since TAD only transport holes, the Alq layer will emit light if the PTCDA layer transports holes to the PTCDA/Alq interface. The characteristic double hump structure, shown in the EL spectrum of Figs. 2(b) and 2(c), indicates that the Alq layer emits light, and the PTCDA layer absorbs part of emitted light. By varying the thickness of the PTCDA (50 and 100 $\mathrm{nm}$ ), we can fit the measured EL spectra with the measured absorption and Alq/TAD EL spectra. The fits (which assume that the top $\mathrm{Al}$ contact is reflecting) indicate that all of the emitted light comes from the Alq layer. This demonstrates that PTCDA transports holes in the direction normal to the molecular planes.

The external quantum efficiency of Alq/TAD LEDs depends on the cathode contact. Typically it is $0.3 \%-0.4 \%$ (photons/electron) with $\mathrm{Al}$ cathodes and increases to more than $0.8 \%$ with bilayer $\mathrm{Li} / \mathrm{Al}$ cathodes. The quantum efficiency of LEDs with a PTCDA hole transporting layer sand-

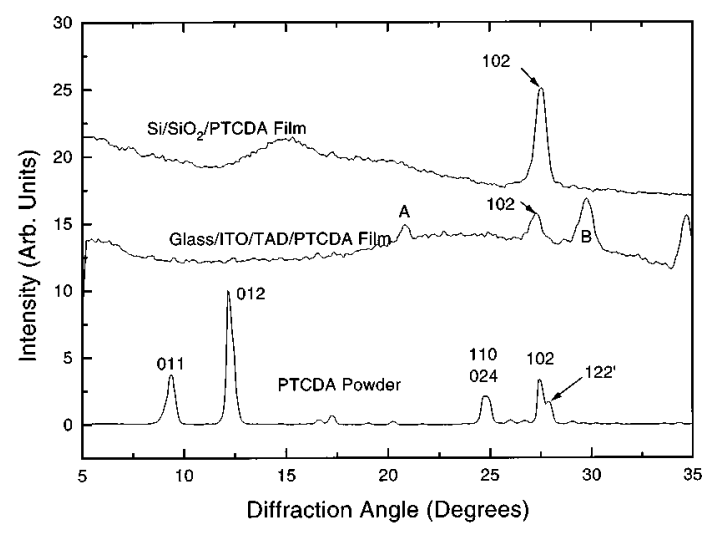

FIG. 4. X-ray diffraction data from the FET structure, LED structure (without Alq and the Al cathode), and PTCDA powder. Both the FET and LED structures have an intensity peak at the (102) plane indicating the PTCDA is parallel to the substrate. The intensity peaks designated as A and B are due to the polycrystalline ITO.

wiched between the Alq and the TAD is generally about an order of magnitude lower than LEDs without PTCDA. This decrease is qualitatively consistent with the findings of Burrows et al., ${ }^{13}$ and may be partly explained by absorption in the PTCDA layer. It is also possible that there are some effects at the PTCDA/Alq interface which lower the quantum yield relative to the TAD/Alq interface.

The device shown in Fig. 2(d) has two Alq layers (one on each side of the PTCDA), and the EL spectrum of this device also has the double hump structure. In fact, the EL spectra of the previous structure with a single emitting layer of Alq and same thickness $(100 \mathrm{~nm})$ of PTCDA has been superimposed to compare the two EL spectra. There is little difference between the two EL spectra. This confirms that thin films of Alq have the ability to transport electrons as well as holes.

Electron transport in PTCDA normal to the plane of the layers was evaluated by studying Al/PTCDA/Alq/TAD LEDs in which the PTCDA is in direct contact with the electron injecting $\mathrm{Al}$ cathode. For the Alq in this device to be able to emit light, the PTCDA must transport electrons. The EL intensity (from Alq) and quantum efficiency were 2 to 3 orders of magnitude lower indicating that most of the injected current is unipolar (hole current). Measurements under vacuum (identical to the conditions used to test the FETs) also do not indicate any appreciable electron transport. This is significant since moisture in the ambient may have impeded electron transport. The fact that no light is seen even when the devices are tested under vacuum is evidence that electron transport is not favored normal to the molecular planes.

\section{Film morphology}

$\mathrm{X}$-ray diffraction measurement were made to ensure that the PTCDA molecules oriented parallel to the substrate in both the FET and LED structures. Low angle x-ray diffraction data, shown in Fig. 4, of thin films of PTCDA in both the FET and LED configuration indicate that the molecular planes of PTCDA lie nearly parallel to the substrate. The 


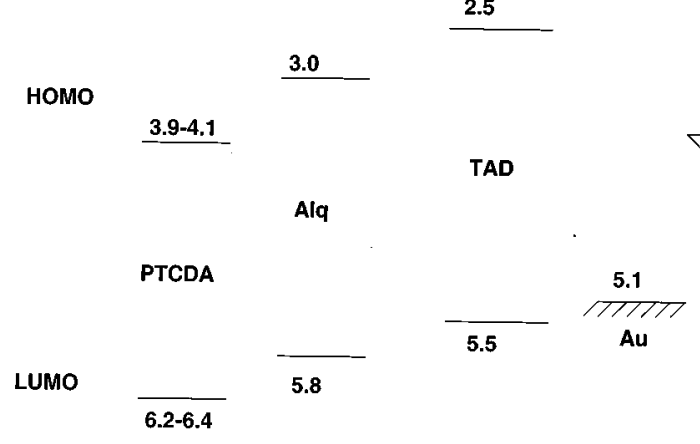

FIG. 5. The HOMO and LUMO energy levels of PTCDA, Alq and TAD and the workfunctions of $\mathrm{Au}$ and $\mathrm{Al}$. The energy values have been assembled from Ref. 20 (PTCDA), Ref. 21 (Alq), and Ref. 22 (TAD).

PTCDA powder gives rise to a spectrum with many peaks corresponding to the different molecular planes. These peak assignments were made with reference to earlier detailed $\mathrm{x}$-ray crystallography data published in Ref. 9. The spectrum from the $\mathrm{Si} / \mathrm{SiO}_{2} / \mathrm{PTCDA}$ sample, the configuration of a FET, has one clear peak near $27.5 \mathrm{deg}$ corresponding to the 102 plane. The x-ray spectrum from glass ITO/TAD/PTCDA sample, the configuration in an LED, has a peak at $27.5 \mathrm{deg}$ (102 plane) as well as 21 and 30 deg (due to the polycrystalline ITO). The presence of the 102 peak and the absence of other peaks in both of the thin film PTCDA samples indicate that the molecules are oriented nearly parallel to the substrate.

Additional characterization of thin film PTCDA morphology was performed with electron diffraction on samples in which PTCDA was deposited on amorphous carbon grids in parallel with the samples used in the x-ray measurements. This technique probes the samples from a direction roughly perpendicular to the plane of the substrate which provides complementary information to the $\mathrm{x}$-ray diffraction data. The electron diffraction data shows a strong signal from the 012 plane and no signal from the 102 plane. When the sample was tilted at an angle $+15 \mathrm{deg}$, the intensity of the diffraction pattern corresponding to the 012 plane reached a maximum, whereas a tilt of $-15 \mathrm{deg}$ produced a reduction in intensity. The electron diffraction data corroborates evidence from the $\mathrm{x}$-ray diffraction measurements that the PTCDA films orient with the molecular planes nearly parallel to the plane of the substrate.

It is important to reiterate that the structural characterization described above was done on PTCDA films deposited under the similar conditions to those used in the device studies. This is significant since early work by Forrest $e t$ al. have shown that the preferential orientation of PTCDA depends on the deposition conditions. ${ }^{12}$ If this is indeed the case, we have no way of knowing a priory if the structure of our PTCDA films are similar to those reported in the literature. Thus, our structural characterization assumes vital importance since the important conclusions depend on the molecular orientation in the two device structures (FET and LED) we employ. More recent structural characterization of PTCDA films grown on Au substrates by Fenter et al. indicate that PTCDA films can be grown quasiepitaxially under the right conditions resulting in highly ordered films. ${ }^{19}$

\section{Energy levels}

Let us now consider the relevant energy levels in the materials considered above, and how these could affect transport properties. The energy levels of the highest unoccupied and the lowest unoccupied molecular orbitals (HOMO and LUMO) are shown in Fig. 5. It is quite evident from the data depicted in Fig. 5 that the barrier to electron injection from the $\mathrm{Al}$ cathode $(\Phi=4.1 \mathrm{eV})$ to the PTCDA (LUMO level $\sim 3.9-4.1 \mathrm{eV}$ ) is small. Despite this favorable energy level alignment, there is very little electron transport through the PTCDA layer as the LED data described above indicate. In the case of the field-effect transistor, there is a $\sim 1 \mathrm{eV}$ barrier between the Au source/drain (S/D) electrodes and the LUMO/HOMO of PTCDA. Unlike the case in organic LEDs, this does not present a serious problem because the FET channel becomes highly conducting in the on state because of the large induced charge density. $C_{60}$ FETs with high mobility have been fabricated with Au S/D electrodes despite the $1.3 \mathrm{eV}$ barrier between the $C_{60}$ LUMO $(3.8 \mathrm{eV})$ and the $\mathrm{Au}$ work function. We have also fabricated $a$-sexithiophene (HOMO energy level=5.2 eV) FETs with Al S/D electrodes $(\Phi=4.1 \mathrm{eV})$. Thus, the energy barrier between the Au contacts and the PTCDA HOMO is unlikely to be the cause of the absence of observable hole transport parallel to the molecular planes. However, the high value of the HOMO $(>6 \mathrm{eV})$ makes it very likely that hole traps at a lower energy prevent the formation of a hole accumulation layer in the FETs.

\section{SUMMARY}

The above data and discussion illustrate the complexity of transport in a molecular thin film. The transport properties depend on a number of factors including molecular ordering and orbital overlap, molecular orbital energy levels, and the nature of the ambient. Nevertheless, it is apparent from our study that hole transport dominates in the direction normal to the molecular planes (where the $\pi$ orbital overlap is high) while along the molecular planes PTCDA behaves as a quasitwo-dimensional electron transporter (in vacuum). This work also illustrates the utility of device measurements in characterizing transport in such materials.

\section{ACKNOWLEDGMENTS}

The authors wish to thank R. C. Dynes and L. J. Rothberg for helpful discussions and I. Brener and D. Weissman for the photoluminescence measurement.

${ }^{1}$ G. Horowitz et al. Appl. Phys. Lett. 67, 528 (1990); F. Garnier et al. Science 265, 1684 (1994); A. Dodabalapur et al. Science 268, 270 (1995).

${ }^{2}$ Semiconductors and Semimetals, edited by E. Conwell (Academic, Boston, 1988), Vol. 27.

${ }^{3}$ H. Mohwald, D. Haarer, and G. Castro, Chem. Phys. Lett. 32, 433 (1975).

${ }^{4}$ N. Karl and J. Ziegler, Chem. Phys. Lett. 32, 438 (1975).

${ }^{5}$ D. Massa and N. Karl, Mol. Cyst. Liq. Cryst. 95, 93 (1989).

${ }^{6}$ L. Torsi, A. Dodabalapur, L. J. Rothberg, A. Fung, and H. E. Katz, Science, 2721462 (1996). 
${ }^{7}$ C. W. Tang, S. A. VanSlyke, and C. H. Chen, J. Appl. Phys. 65, 3610 (1989).

${ }^{8}$ A. Dodabalapur, L. J. Rothberg, and T. M. Miller, Electron. Lett. 30, 1000 (1994).

${ }^{9}$ A. J. Lovinger et al. J. Appl. Phys. 55, 476 (1984).

${ }^{10}$ C. Ludwig et al. Zeitschrift Fur Physik B 93, 365 (1994).

${ }^{11}$ The detection of small hole currents in dominantly $n$-channel FETs has been analyzed in detail by our group in A. Dodabalapur et al. Appl. Phys. Lett. 68, 1108 (1996).

${ }^{12}$ S. R. Forrest, M. L. Kaplan, and P. H. Schmidt, J. Appl. Phys. 55, 1492 (1984); J. Appl. Phys. 56, 543 (1984).

${ }^{13}$ P. E. Burrows and S. R. Forrest, Appl. Phys. Lett. 64, 2285 (1994).

${ }^{14}$ D. Y. Zang, F. So, and S. R. Forrest, Appl. Phys. Lett. 59, 823 (1991).

${ }^{15}$ D. Y. Zang, Y. Q. Shi, F. So, S. R. Forrest, and W. H. Steier, Appl. Phys. Lett. 58, 562 (1991).
${ }^{16}$ D. Ammermann, C. Rompf, and W. Kowalski, Jpn. J. Appl. Phys. 34, 1293 (1995).

${ }^{17}$ H. Fuchigami, S. Tanimura, Y. Uehara, T. Kurata, and S. Tsunoda, Jpn. J. Appl. Phys. 34, 3852 (1995).

${ }^{18}$ R. G. Kepler et al. Appl. Phys. Lett. 66, 3618 (1995).

${ }^{19}$ P. E. Fenter, P. E. Burrows, P. Eisenberger, and S. R. Forrest, J. Cryst. Growth 152, 65 (1995).

${ }^{20}$ Y. Hirose, W. Chen, E. I. Haskal, S. R. Forrest, and A. Kahn, Appl. Phys. Lett. 64, 3482 (1994); N. Karl and N. Sato, Mol. Cyst. Liq. Cryst. 218, 79 (1992).

${ }^{21}$ C. Hosokawa, H. Higashi, and T. Kusumoto, Appl. Phys. Lett. 62, 3238 (1993).

${ }^{22}$ C. Adachi, K. Nagai, and N. Tamoto, Appl. Phys. Lett. 66, 2679 (1995). 